\title{
Model could aid emergency response planning for foot-and-mouth disease outbreaks
}

by Mimako Kobayashi, Richard E. Howitt and Tim E. Carpenter

Infectious animal diseases are an ever-present threat to intensive livestock production. We analyzed control technology for foot-and-mouth disease (FMD) in a livestock-intensive region of the Central Valley, using a previously developed, numerical, optimal disease-control model. We found that the alternative FMD controls we studied (early detection, herd depopulation and vaccination) can be partially substituted for one another (substitutability) without substantially changing outbreak costs. This information can be used to develop effective and efficient policies to prepare for an FMD outbreak in California.

The risk of infectious animal dis1 eases is an inherent and unavoidable problem in commercial livestock production. On the supply side, as production geographically concentrates and intensifies, both the risks and consequences of disease outbreaks increase. On the demand side, dependence on access to international markets increases outbreak costs, because importing countries close their markets during and in the aftermath of a disease outbreak. Because animal diseases can spread from farm to farm, a farm's actions to prevent and control diseases have positive spillover effects or "externalities" by reducing the probability that other farms are infected (Sumner et al. 2005). Economic theory tells us that in the presence of externalities, the private sector alone will not make sufficient investments in disease prevention and control. Therefore, the public sector has an important role in ensuring that mechanisms are in place to manage disease outbreaks in intensive livestockproduction systems.

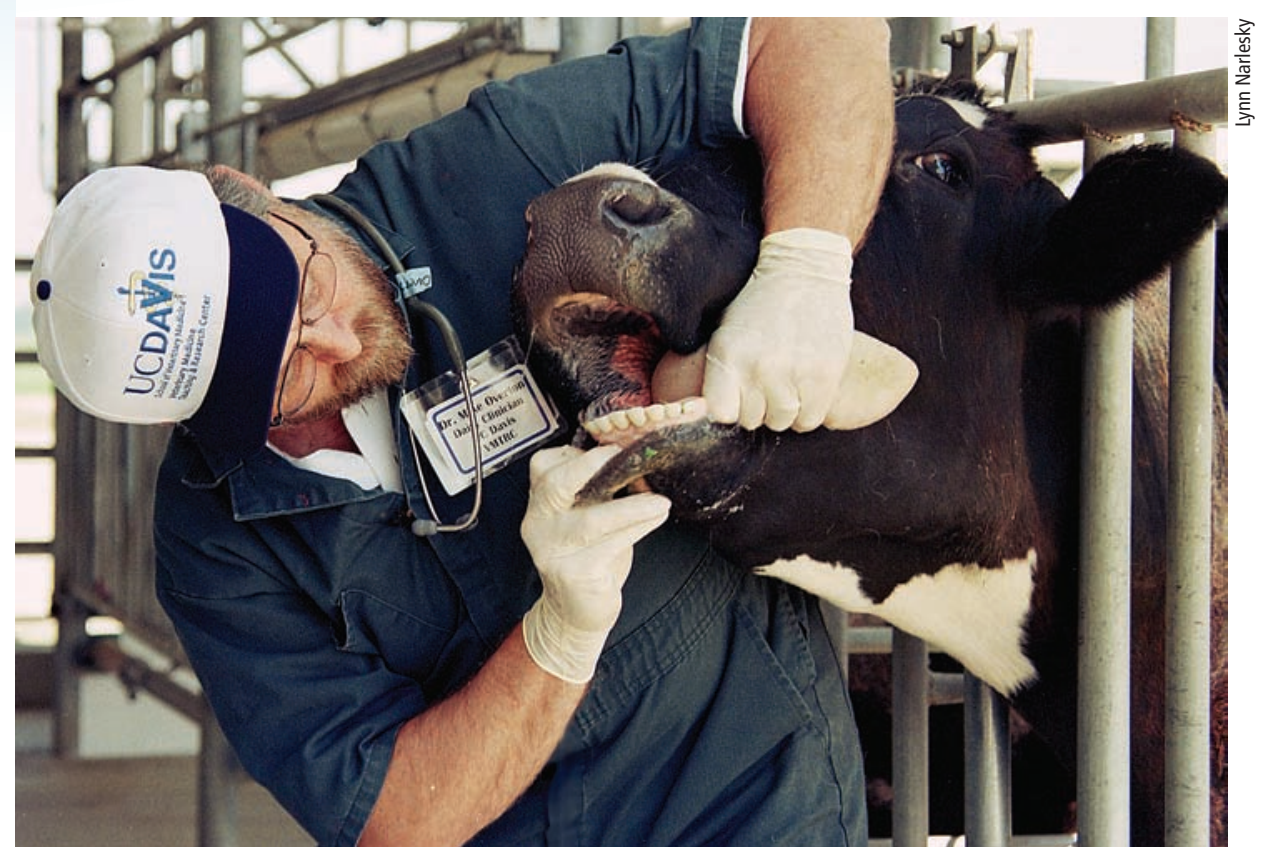

Using models to plan for outbreaks of infectious animal disease helps public policymakers to allocate resources more effectively. Michael Overton checked a healthy dairy cow for foot-andmouth disease at UC Davis.

Public planning for potential emergency situations entails making rules and guidelines about how to respond when such events occur. The response is limited by the availability of resources. For some resources, procurement or construction is necessary before emergency situations occur. Effective planning also involves prior investments in response capacities, which determine the scale of response measures. We analyze how such investment decisions can be made when different types of response measures interact in a nonlinear way. We demonstrate that knowledge about substitutability among response measures (in this case the ability to increase some measures and decrease the others without changing the overall outbreak costs) enables the decision-maker to prioritize and target investments.

\section{Emergency response to outbreaks}

Emergency responses to an infectious livestock-disease outbreak involve several dimensions. Measures should be taken to (1) expedite the initial response, which may be partially achieved by early detection of cases and communication with decision-makers, (2) reduce the disease's spread and (3) enable a swift recovery. There are alternative approaches, however, and the process by which disease-control efforts interact is usually nonlinear and complex. For example, emergency vaccinations and bans on the movement of infected animals limit a disease's spread; but in order to find an efficient combination of the two measures, the decision-maker requires information about how effectively each measure works and whether the two measures are substitutable in achieving an overall objective. During the planning process, information about the relative effectiveness of alternative measures can be compared with their costs to determine how resources should be allocated.

\section{Potential FMD outbreak}

We analyzed a response-capacity investment problem for a potential outbreak of an exotic livestock disease in California, foot-and-mouth disease (FMD). FMD is highly contagious and if it were to infect livestock, the economic 
consequences could be substantial and extensive (Ekboir 1999; Paarlberg et al. 2003). Although the United States has been free of FMD since 1929, public and private preparations for a potential outbreak are important to safeguard intensive livestock-production systems in California (CDFA 2006b) and elsewhere. During an FMD outbreak among livestock herds, response measures typically include (1) movement restrictions on animals, people and vehicles, (2) herd depopulation and (3) emergency vaccination (this may not, however, be available in the United States). Active surveillance of livestock operations allows early detection of the first case and limits subsequent damage. Due to the disease's fast-spreading nature, government regulators and the livestock industry can not build or expand the infrastructure/capacity of these activities while an outbreak is in progress, so careful planning is required before a disease outbreak occurs.

Central Valley study area. We analyzed this problem for a three-county (Fresno, Kings and Tulare) region in the Central Valley. In 2002, the region housed about 1.8 million head of FMDsusceptible livestock (cattle, hogs, sheep and goats) (USDA-NASS 2004) (table 1). More than half (54\%) were dairy cattle, $31 \%$ beef cattle, $11 \%$ pigs and $4 \%$ sheep
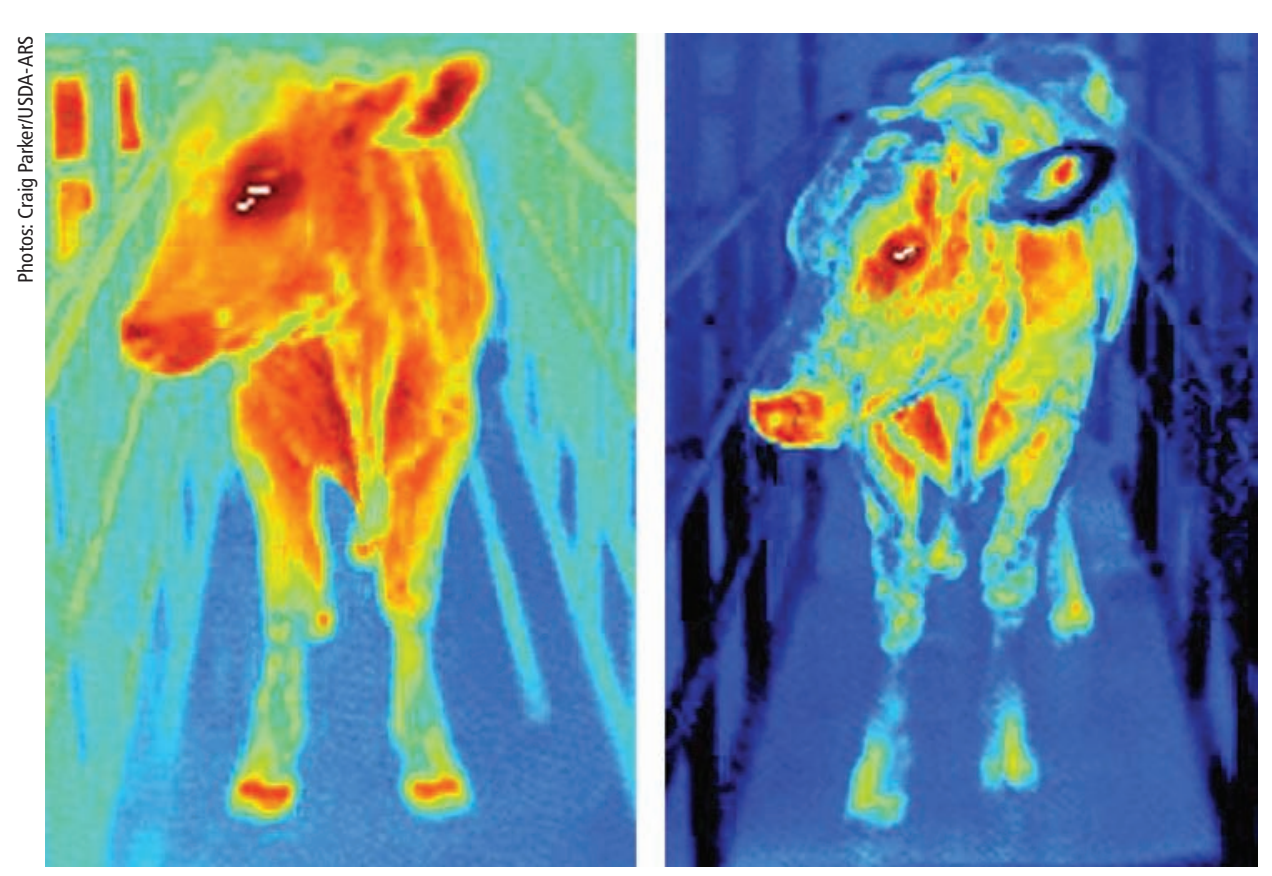

Foot-and-mouth disease is highly contagious and difficult to detect in its early stages. Left, an

infrared image of an infected cow; the red color in the hooves indicates heat. Right, a healthy cow. and goats, and less than $1 \%$ were backyard animals. The region is characterized by a concentrated distribution of large-scale dairy operations, accounting for $43 \%$ of California's milk production and $58 \%$ of its cattle production in output value in 2005 (CDFA 2006a). Given the high asset values of dairy cattle (table 1) and the importance of dairy production to California's agricultural economy ( $\$ 5.2$ billion or $14 \%$ of total agricultural output in 2005 [CDFA 2006a]), the region receives much of the state's FMD preparation efforts (Richard Breitmeyer, California state veterinarian, personal communication).

Optimization model. We derived the technical interactions of FMD response measures in California using a previously developed, numerical, dynamic optimization model (Kobayashi et al. 2007a). The optimization model finds FMD control strategies that minimize the region, given user-specified levels of resource availability (response capacity). The specification and parameterization of the optimization model were based on a detailed, spatially explicit epidemiological simulation model for FMD (Bates et al. 2003) developed for the three-county region. In this study, we considered surveillance, carcass disposal and vaccination capacities. By the total direct costs of an outbreak for varying the response capacity levels, we

TABLE 1. Primary livestock industry structure in
three-county California region

* na $=$ not applicable; we assume that animals are moved to a non-sales-yard premises at the end of each day when FMD control measures are implemented.

Source: Parameters from optimal FMD control model by Kobayashi et al. (2007a). Herd no.: September 2000 surve Bates, Thurmond, et al. 2003; herd size and livestock population, USDA-NASS 2004; livestock herd value, USDANASS 2005, USDA 2005.

analyzed how changes in the relative availability of response measures affect the overall outcome of FMD control.

Pre- and post-outbreak responses.

Planning for and investing in the capacity to prevent diseases can also reduce the probability that a disease will be introduced. Since Elbakidze and McCarl (2006) studied the problem of allocating resources between prevention and postevent activities, we focused on the problem of capacity investment decisions in post-outbreak activities. Moreover, optimal capacity investments should reflect the probability of outbreaks. Although some estimates are available at the national level (USDA-APHIS 1995), to our knowledge, probability estimates of FMD virus introduction in California are unavailable. We discuss the relative, not absolute, capacity of different response measures without making assumptions about the probabilities of FMD introduction.

\section{Measures to control FMD}

FMD is a highly contagious disease affecting cloven-hoofed animals such as cattle, pigs, sheep, goats and deer, but not humans. It results in increased mortality in young animals and reduced productivity in mature animals (Hyslop 1970). Early detection and control, which includes culling herds that are infected or potentially infected, is important to limit the disease's spread and the duration of an epidemic as well as enable the reestablishment of trade with FMD-free nations (OIE 2008). 
FMD is difficult to detect initially and a delay in implementing control policies is almost inevitable. An animal infected by the FMD virus becomes infectious after a few days (the latent period), but clinical signs, if any, appear a few days after the subclinically infectious period. Moreover, clinical signs on an individual animal can be subtle and may not be noticed immediately or may be confused with other diseases. Because of its high infectiousness, the disease is likely to have spread to other herds by the time the first case is detected. In the 2001 FMD outbreak in the United Kingdom, the estimated detection lag between the initial infection and confirmation was 21 days, and the disease spread to at least 57 herds (Gibbens and Wilesmith 2002). Surveillance activities for early disease detection are an important investment option to prepare for a potential FMD outbreak.

Movement restrictions. Upon detection of the first case, movement restrictions on animals, vehicles and people would be imposed within a specified geographical area. In California, the restrictions would likely be imposed statewide initially, with the area subsequently reduced as more accurate information about the extent of the disease's spread was obtained (Speers et al. 2004).

Eradication. Subsequent eradication policy would be applied to all herds in which clinically infected animals had been found. Additional herds might be preemptively depopulated if they were considered potentially infected. In the 2001 U.K. outbreak, preemptive depopulation was applied to herds that were contiguous to, or had known recent contacts with, confirmed infected herds. In total, more than 4 million animals were slaughtered for disease control purposes, of which about two-thirds later turned out to be uninfected (NAO 2002). In addition, 2.3 million animals were slaughtered for animal welfare reasons, because they could not be marketed or feeds could not be procured due to movement restrictions (NAO 2002).

Vaccination. Emergency vaccination may limit the disease's spread by reducing shedding in infected animals and the exposure risk in susceptible herds. However, testing technology and its ability to discern vaccinated animals from FMD-infected ones (Breeze 2004)
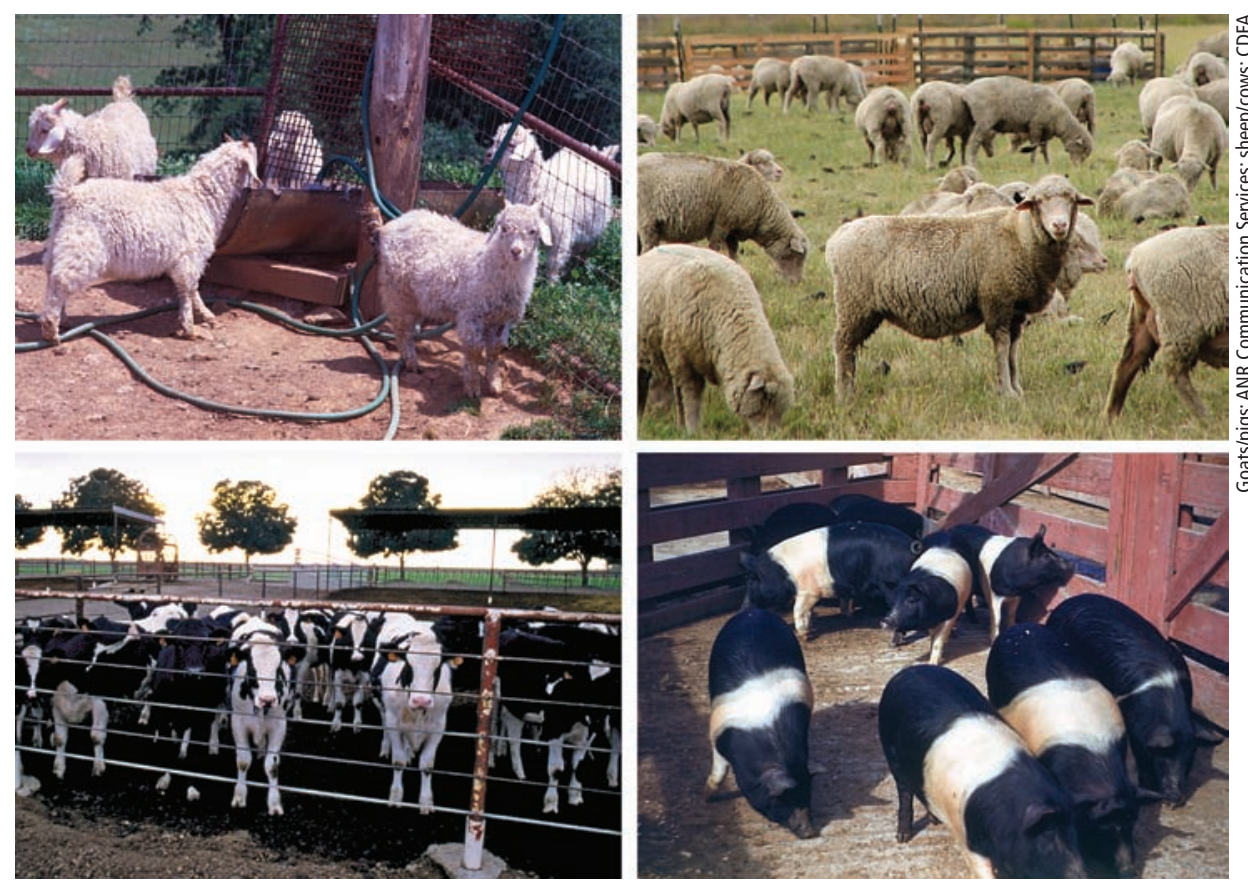

A model was used to compare the benefits of control strategies such as vaccination, surveillance and carcass disposal. Cloven-hoofed animals - including, clockwise from top left, goats, sheep, pigs and cows - are affected by foot-and-mouth disease, but not humans.

may not be accepted by trading partners, and international trade restrictions may nonetheless result. Even after an outbreak is contained, a country that has used the FMD vaccine may be differentiated from countries without vaccination and continue to face trade restrictions. An FMD-free country can officially gain an FMD-free-withoutvaccination status by slaughtering all FMD-vaccinated animals (OIE 2005). Facing an FMD outbreak, a previously FMD-free country has three options: (1) no vaccination; (2) vaccination without slaughtering vaccinated animals ("vaccinate-to-live"), which possibly triggers trade restrictions; and (3) vaccination and then slaughter of vaccinated animals ("vaccinate-to-kill").

In the United States, decisions about the use of emergency vaccination are made at the federal level by the U.S. Department of Agriculture on a case-bycase basis. Therefore, in the absence of a specific case, the choice of vaccination options is unknown. In our three-county study region, large-scale dairy herds are expected to have disproportionately high infection rates due to the frequent movement of animals, people and vehicles to and from these operations (Bates et al. 2001). Given the high asset value of these dairy herds, local regulatory veterinarians prefer the vaccinate-to-live option to protect the herds first from in- fection, and then from depopulation ( $\mathrm{R}$. Breitmeyer, California state veterinarian, personal communication). Uncertainty surrounding federal vaccination policy poses a challenge to California's FMD preparation efforts.

\section{Optimal FMD control model}

Kobayashi et al. (2007a) developed a numerical optimization model of FMD control and parameterized it for the three-county region of California with 2,243 herds (table 1). A set of 36 disease-transmission parameters was estimated using output generated by a prior epidemic simulation model (Bates, Thurmond, et al. 2003), where herdto-herd infection was explicitly modeled as a result of direct (animal) and indirect (vehicles and people) contact between herds and local-area spread. The 36 parameters predict the aggregate effects of the three modes of disease transmission.

While disease dynamics are initiated by specifying one index (initial infection) herd, daily disease spread is affected by control measures in the model. First, the depopulation of infected herds prevents further spread of disease by containing it at the source. Subsequent carcass disposal and cleaning and disinfection of the premises may be considered as recovery measures. However, a delay in carcass disposal can cause 


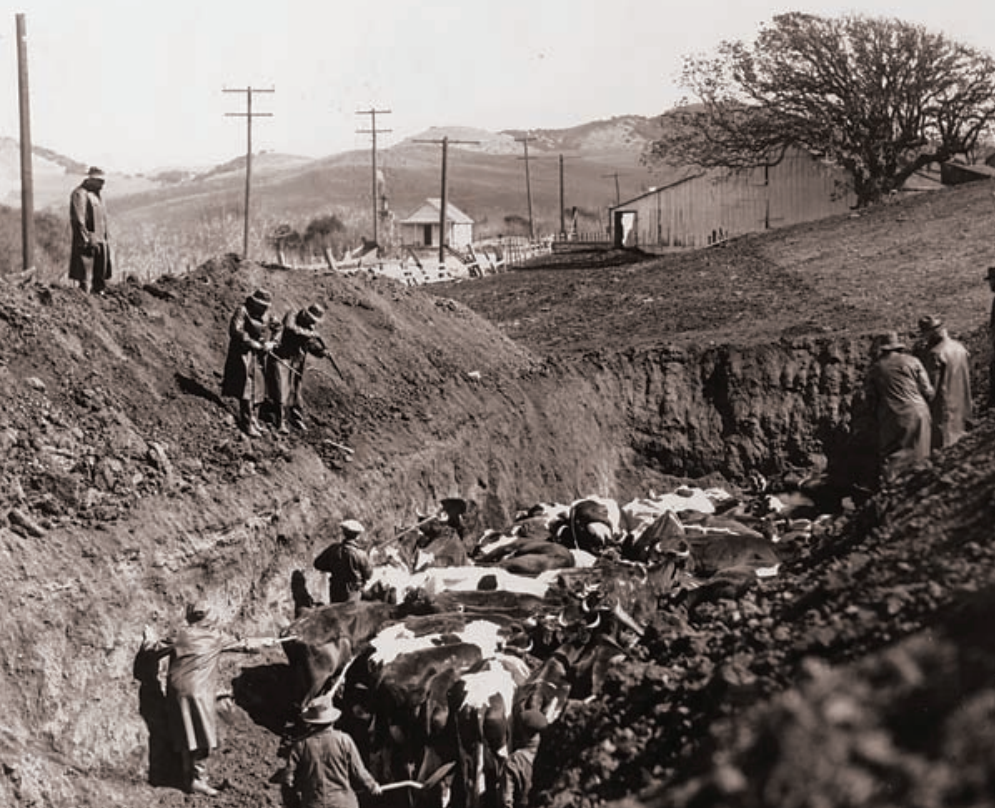

The last California outbreaks of foot-and-mouth disease were in 1924 and 1929. In 1924, a Southern California dairy herd was killed and buried to prevent further spread of the virus. depopulation and vaccination levels. Outbreak costs includes those for implementing controls (depopulation, vaccination and movement restrictions) and the value of livestock herds depopulated for disease control (Kobayashi et al. 2007a). Caveats on the cost specifications are that we did not consider international trade consequences or linkage effects with nonlivestock sectors (such as tourism). Similarly, even though an outbreak may expand farther, we secondary infections of other herds, so the effectiveness of herd depopulation in the model depends on the capacity to dispose of carcasses. Second, emergency vaccination limits further spread to susceptible herds, although the daily availability of FMD vaccine would affect the scale of vaccination.

Movement restrictions on animals, vehicles and people further reduce the disease's spread; these are accounted for by lower disease-transmission parameters in the model. The second set of 36 disease-transmission parameters was estimated using data generated by the simulation model, with equivalent movement-restriction specifications. The estimated parameters were reduced by $55 \%$ to $82 \%$, except for sales yards, which were reduced by $100 \%$ since they would be closed immediately upon detection of the disease in the region (Kobayashi et al. 2007a).

Finally, a delay in disease detection would also affect disease dynamics and the duration and size of an outbreak. Measures that allow early disease detection, such as routine active surveillance, are another possible area of capacity investment. Kompas et al. (2006) also investigate optimal local surveillance levels in preparation for an FMD outbreak in the United States.

Cost assumptions. Given the disease spread parameters and capacity specifications for carcass disposal, vaccination and disease detection, the optimization model minimizes outbreak costs by choosing daily herd
Response capacities. A range of response capacities was implemented in the model. Surveillance investment levels were measured in terms of the time taken for the first case to be diagnosed - between 7 and 21 days after initial infection - assuming that, with experience, the disease would be found sooner than the 21 days it took in the U.K. 2001 outbreak. For carcass disposal capacity, without estimates of current capacity in the California threecounty region, we considered levels ranging from 1,000 to 20,000 head per day. (Limitations in the region's carcassrendering capacity were confirmed when a heat wave increased mortality among dairy cattle in summer 2006 [Souza 2006].) While a wide variety of alternative methods are available, such as burial, incineration and composting (NABCC 2004), the actual choice would be based on relative costs, and public health and environmental impacts and regulations. Carcass disposal by onfarm pyre and burial during the 2001 U.K. outbreak raised concerns about air and groundwater pollution (NAO 2002). Should an FMD outbreak occur in the United States, carcass disposal procedures would face close scrutiny (NABCC 2004).

We first implemented the novaccination policy, since the availability of this option is uncertain. Then we implemented the vaccinate-to-live policy at various vaccine availability levels. Currently in the United States, the FMD vaccine stockpile is controlled at the federal level and a state cannot inde-

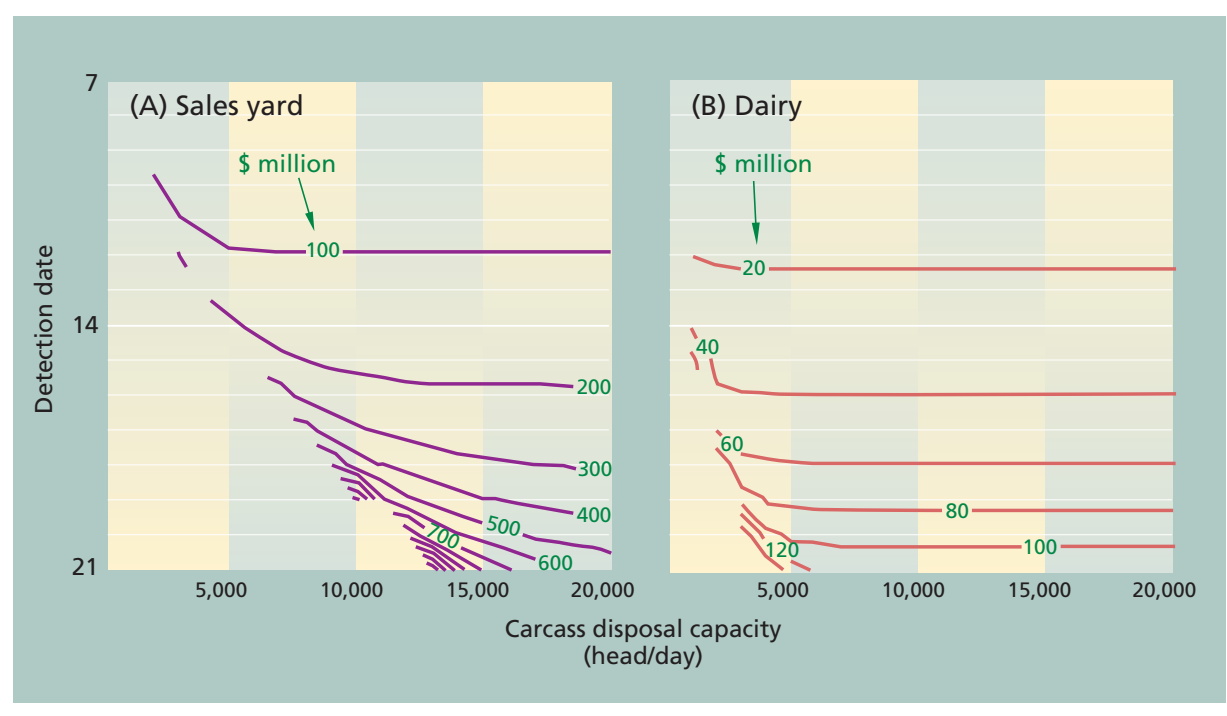

Fig. 1. Iso-cost curves under no-vaccination policy, showing combinations of detection date and carcass disposal capacity that attain the same overall cost for (A) sales yard and (B) dairy. Moving toward the bottom left corresponds with tighter capacities, increasing total outbreak cost. 
pendently invest in increased vaccine availability. We used federal estimates (Speers et al. 2004), and considered one to five times the estimates as the region's vaccine availability. (The vaccine is strain-specific, posing a limitation in capacity building through stockpiling.) Speers et al. (2004) estimated that 250,000 doses would arrive 4 days after the first case was diagnosed; after 4 more days, 500,000 doses would arrive; and a week later and every week after that, a million doses would arrive.

Previous studies have found that the size of a potential FMD outbreak in this region would significantly depend on where the index case occurred (Bates, Carpenter, et al. 2003; Kobayashi et al. 2007b). An outbreak would be largest if a sales yard is the index case, followed by a dairy herd. Most results that we show were generated by specifying a sales yard as the index case, representing the worst-case scenario.

\section{Substitutability between controls}

The nature of FMD-control technology is presented by curves with constant costs over different sets of parameters (iso-cost curves) (fig. 1). The iso-cost curves illustrate how different combinations of detection date (days elapsed since initial infection, ranging from 7 to 21 days) and carcass disposal capacity ( 0 to 20,000 head per day) achieve different overall cost levels, when vaccination is not available and either a sales yard (fig. 1A) or a dairy herd (fig. 1B) is the index case. Downwardsloping iso-cost curves show that surveillance and carcass disposal capacity can be substituted without changing the outbreak costs. For example, an outbreak will cost $\$ 200$ million with detection on day 14 and carcass disposal of about 5,000 head per day, but the same cost can be achieved with a detection delay of 1 day (detection on day 15) and an additional carcass disposal capacity of 2,500 head (fig. 1A).

Compared to the situation where a sales yard is the index case (fig. 1A), costs associated with each capacity combination are much smaller when a dairy herd is the index case (fig. 1B), because an outbreak that starts on a dairy farm would be smaller. Moreover, except with carcass disposal capacity of less than about 6,000 head per day, the iso-cost curves are completely flat, indicating that additional carcass disposal capacity would not contribute to a reduction in overall costs. This also implies that in choosing absolute levels of capacity investments, the distribution of expected outbreak size - in addition to the probability and frequency of outbreaks - should be considered.

\section{Vaccinate-to-live policy}

While current U.S. federal policy may not be favorable toward the use of emergency FMD vaccinations, California veterinary officials generally favor a relaxed vaccination policy. We implemented the "vaccinate-to-live" option to analyze its impacts on overall costs, and assumed a sales yard as the index case.

The iso-cost curves demonstrate substitutability between carcass disposal capacity and vaccine availability (ranging from one to five times the current available estimate) when the detection date is held constant at days 21, 18 and 14 (figs. 2A-C). As the disease is detected sooner, the iso-cost curves become steeper, indicating a smaller role of vaccine availability for a given carcass disposal capacity. For example, when detection is on day 21 (fig. 2A), with the current vaccine availability estimates and disposal capacity of 10,000 head per day, doubling vaccine availability would reduce costs by about $\$ 40$ million (from $\$ 540$ million to $\$ 500$ million), whereas when detection is on day 18 (fig. 2B), the same increase in vaccine availability would reduce costs by about $\$ 20$ million (from $\$ 320$ million to $\$ 300$ million).

The iso-cost curves are fairly flat for substitutability between carcass disposal

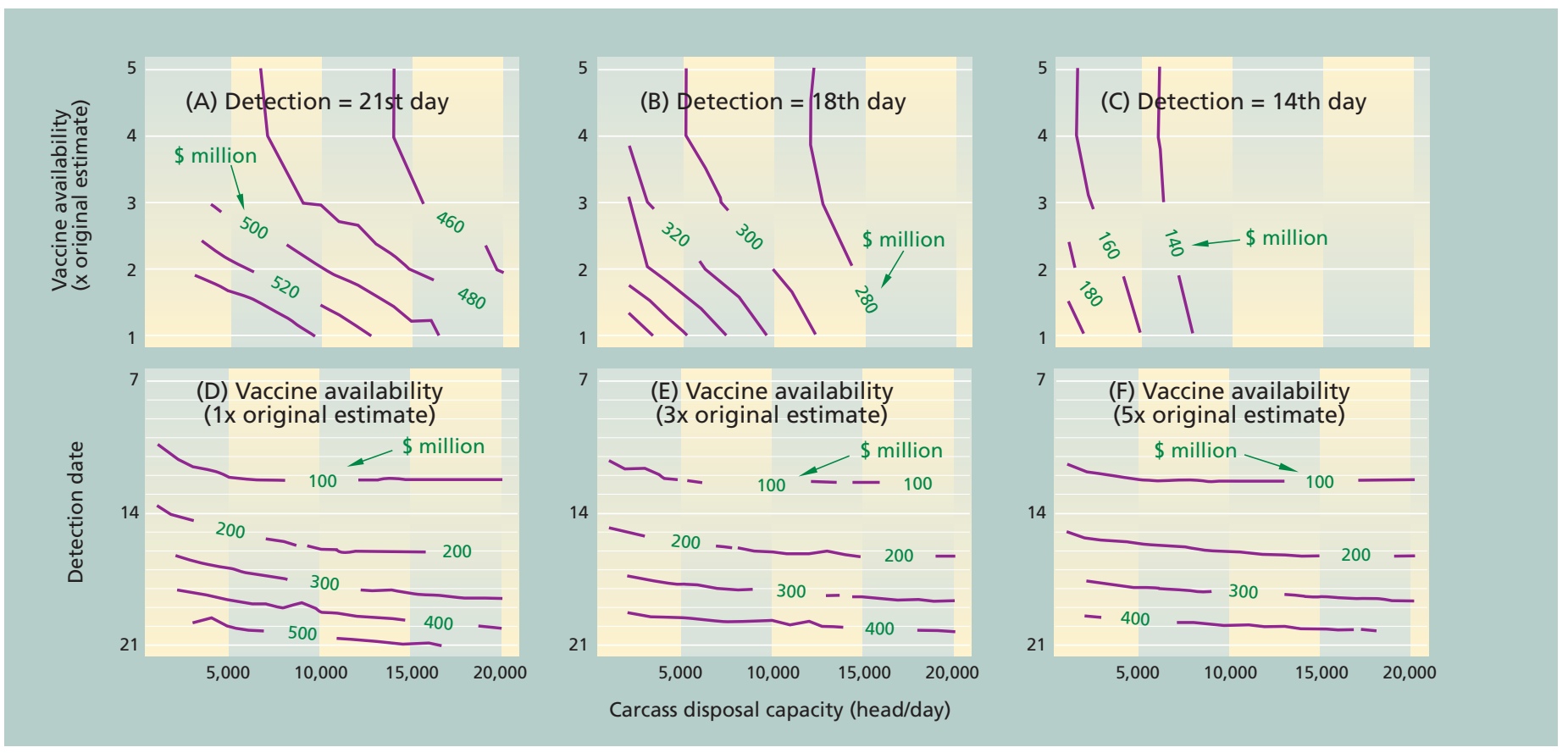

Fig. 2. Iso-cost curves under vaccinate-to-live policy (index case = sales yard), showing combinations of two capacities that attain the same overall cost levels while the third capacity is held constant. 
capacity and surveillance when vaccine availability is held constant (fig. 2D-F). If the disease is detected sufficiently early, it would not spread as widely, so a small carcass disposal capacity would be sufficient to dispose of infected animals and limit further disease spread. However, to the degree that initial detection is delayed, a larger disposal capacity is required to keep outbreak costs low. The iso-cost curves are flatter for higher vaccine availability, suggesting that the role of carcass disposal diminishes due to substitutability between vaccination and depopulation (figs. 2A-C).

When disease detection is sufficiently early, the iso-cost curves for the no vaccination (fig. 1A) and vaccinate-to-live (fig. 2D) policies are similar. However, as disease detection is delayed, the iso-cost curves for no vaccination (fig. 1A) are steeper and associated with higher overall costs than those for the vaccinate-to-live policy (fig. 2D), suggesting that without vaccination, delayed detection would re-

\section{References}

Bates TW, Carpenter TE, Thurmond MC. 2003. Benefit-cost analysis of vaccination and preemptive slaughter as a means of eradicating foot-and-mouth disease. Am J Vet Res 64:805-12.

Bates TW, Thurmond MC, Carpenter TE. 2001. Direct and indirect contact rates among beef, dairy, goat, sheep and swine herds in three California counties, with reference to control of potential foot-and-mouth disease transmission. Am J Vet Res 62:1121-9.

Bates TW, Thurmond MC, Carpenter TE. 2003. Description of an epidemic simulation model for use in evaluating strategies to control an outbreak of footand-mouth disease. Am J Vet Res 64:195-204

Breeze R. 2004. Agroterrorism: Betting far more than the farm. Biosecur Bioterror: Biodef Strat Pract Sci 2:251-64

[CDFA] California Department of Food and Agriculture. 2006a. California Agricultural Resource Directory 2006. Sacramento, CA. www.cdfa.ca.gov/files/pdf/ card/AgResDirEntire06.pdf.

CDFA. 2006b. Foreign Animal Disease Emergency Response Executive Overview. Sacramento, CA. www. cdfa.ca.gov/ahfss/Animal_Health/pdfs/Overview_FAD_ Response_1.pdf

Ekboir JM. 1999. Potential Impact of Foot-and-Mouth Disease in California: The Role and Contribution of Animal Health Surveillance and Monitoring Services. UC Agricultural Issues Center, Davis, CA.

Elbakidze L, McCarl B. 2006. Animal disease preevent preparedness versus post-event response: When is it economic to protect? J Agr Appl Econ $38(2): 327-36$

Gibbens JC, Wilesmith JW. 2002. Temporal and geographical distribution of cases of foot-and-mouth disease during the early weeks of the 2001 epidemic in Great Britain. Vet Rec 151:407-12.

Hyslop NStG. 1970. The epizootiology and epidemiology of foot-and-mouth disease. Adv Vet Sci Compar Med 14:261-307.

Kobayashi M, Dickey BF, Carpenter TE, Howitt RE. 2007a. A dynamic optimal disease control model for foot-and-mouth disease. I. Model description. Prev Vet Med 79:257-73.

Kobayashi M, Dickey BF, Carpenter TE, Howitt RE. 2007b. A dynamic optimal disease control model for foot-and-mouth disease. II. Model results and policy implications. Prev Vet Med 79:274-86
Kompas T, Che TN, Ha PV. 2006. An Optimal Surveillance Measure Against Foot-and-Mouth Disease in the United States. Crawford School of Economics and Government, Australian National University, Canberra, Australia. Working Paper 06-11.

[NABCC] National Agricultural Biosecurity Cente Consortium. 2004. Carcass Disposal: A Comprehensive Review. Kansas State University; Carcass Disposal Working Group for the USDA-APHIS. http:// fss.k-state.edu/FeaturedContent/CarcassDisposal/ CarcassDisposal.htm.

[NAO] National Audit Office. 2002. The 2001 Outbreak of Foot and Mouth Disease. London, UK. www.nao. org.uk/publications/nao_reports/01-02/0102939.pdf

[OIE] World Organisation for Animal Health. 2008 Terrestrial Animal Health Code. Article 8.5.1. Paris, France. www.oie.int/eng/normes/mcode/en chapitre_1.8.5.htm\#rubrique_fievre_aphteuse.

Paarlberg PL, Lee JG, Seitzinger AH. 2003. Measuring welfare effects of an FMD outbreak in the United States. J Ag App Econ 35:53-65.

Souza C. 2006. Heat wave takes toll on livestock crops. California Farm Bureau Federation. AgAlert, Aug. 2. www.cfbf.com/agalert.

Speers R, Jonas D, Giovachino M, et al. 2004. Analysis and Recommendations from Operation "Aphtosa." IPR 11239. California Department of Food and Agriculture, Sacramento, CA; CNA Corporation, Alexandria, VA.

Sumner DA, Bervejillo JE, Jarvis LS. 2005. Public policy, invasive species and animal disease management. Int Food Agribus Manage Rev 8:78-97.

[USDA] US Department of Agriculture. 2005. Meat Animals Production, Disposition, and Income 2004 Summary, April 2005. http://usda.mannlib.cornell.edu/ reports/nassr/livestock/zma-bb/meat0405.pdf

[USDA-APHIS] USDA Animal and Plant Health Inspection Service. 1995. Risk assessment of the practice of feeding recycled commodities to domesticated swine in the U.S. Washington, DC.

[USDA-NASS] USDA National Agricultural Statistics Service. 2004. 2002 Census of Agriculture. Washington, DC. www.agcensus.usda.gov/Publications/2002/ index.asp.

USDA-NASS. 2005. California Agricultural Statistics 2004. www.nass.usda.gov/pub/nass/ca/ AgStats/2004cas-all.pdf. quire compensation for a much larger carcass disposal capacity.

\section{Flexible disease-control technology}

We found technical flexibility in FMD control, in that surveillance, herd depopulation and vaccination activities can be substituted without changing the overall level of outbreak costs. The iso-cost curves clearly illustrate that substitutability between capacities exists for a certain capacity range, and the range depends on the index case.

Flexibility in control technology gives decision-makers choices in how to build capacity to control a livestock disease outbreak. With flexibility, it is possible to choose capacity combinations with lower investment costs or combinations that attain higher environmental or public health standards. Without flexibility, possible capacity combinations are determined entirely by the technology, and investments could be costlier. The iso-cost curves also show that decision-makers have a choice between achieving a low outbreak cost with high capacities (high investment costs) and achieving a high outbreak cost with low capacities (low investment costs). Balancing pre-event (investment) and post-event (control) cost trade-offs is a key element of emergency response planning and management, and the information generated in this study is useful for evaluating such decision problems.

By combining knowledge of epidemiology and economics, valuable information with direct policy implications can be obtained. We encourage continued collaboration between the biophysical sciences and economics, in order to promote efficient preparation and decision-making for potential disasters.

M. Kobayashi is Research Assistant Professor, Department of Resource Economics, University of Nevada, Reno; R.E. Howitt is Professor and Chair, Department of Agricultural and Resource Economics, UC Davis; and T.E. Carpenter is Professor and Co-Director, Center for Animal Disease Modeling and Surveillance, Department of Medicine and Epidemiology, School of Veterinary Medicine, UC Davis. The authors thank the three anonymous referees for helpful comments. This study was supported by the Department of Homeland Security and the National Center for Foreign Animal and Zoonotic Disease Defense. 